\title{
Eclipsing Spectroscopic Binaries in the SMC
}

\author{
Ron W. Hilditch \\ School of Physics and Astronomy, University of St Andrews, North \\ Haugh, St Andrews, Fife KY16 9SS, UK \\ Tim J. Harries \\ School of Physics, University of Exeter, Stocker Road, Exeter EX4 4QL, \\ UK \\ Ian D. Howarth \\ Department of Physics and Astronomy, University College London, \\ Gower Street, London WC1E $6 B T$
}

The OGLE survey of the SMC has discovered 1500 eclipsing binaries thereby providing an excellent platform to study the evolution of close binary systems through case A and case B mass-exchange processes. The complementary spectroscopic radial-velocity studies of these binaries are now in progress and are revealing many interesting systems which challenge current theoretical models of close binary star evolution. These studies also provide excellent direct determinations of distances to these binary stars leading to an improved understanding of the mean distance to the SMC and its 3-D structure. Comparisons between these binary-star distances and other methods of determining the mean distance to the SMC will also be made. 